\title{
Off-Diagonal Magnetoimpedance Dependence of Magnetostriction and Anisotropy in Co-Based and Fe-Based Amorphous Ribbons
}

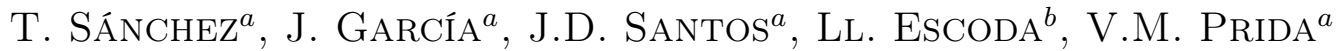 \\ M.L. SÁNCHEZ ${ }^{a}$, J.J. SUÑOL ${ }^{b}$ AND B. HERnANDO ${ }^{a, *}$ \\ ${ }^{a}$ Universidad de Oviedo, Departamento de Física, C/Calvo Sotelo s/n, 33007-Oviedo, Spain \\ ${ }^{b}$ Universitat de Girona, Campus de Montiliví, Edifici PII, Lluis Santaló s/n, 17003-Girona, Spain
}

The object of this work is the comparison of domain structure and off-diagonal magnetoimpedance effect in amorphous ribbons with different magnetostriction coefficient. The $\mathrm{Co}_{66} \mathrm{Fe}_{4} \mathrm{Ni}_{1} \mathrm{Si}_{15} \mathrm{~B}_{14}$ and $\mathrm{Fe}_{80} \mathrm{~B}_{20}$ samples were obtained by melt-spinning. During the quenching procedure a $0.07 \mathrm{~T}$ transverse magnetic field was applied to some of the samples. Domain patterns obtained by the Bitter technique confirm that the differences on the samples are related to the different anisotropy and magnetostriction coefficient, and the quenching procedure. Small changes on the anisotropy distribution and the magnetostriction coefficient can be detected by the off-diagonal impedance spectra as a consequence of the different permeability values of the samples.

PACS numbers: $75.30 . \mathrm{Gw}, 75.50 . \mathrm{Kj}, 75.60 . \mathrm{Ch}$

\section{Introduction}

An important parameter to tailor amorphous ribbons in order to make them suitable for applications is magnetic anisotropy [1]. This is usually induced by thermal treatments that lead to a deterioration of the good mechanical properties of the samples [2]. The small magnetic anisotropies induced by applying a magnetic field during the quenching process have shown not to deteriorate the excellent mechanical properties of as quenched ribbons [3]. The magnetic anisotropy determines the domain structure of the samples [4]. The magnetoimpedance (MI) effect has been shown to be an effective tool in order to investigate the magnetic properties of soft magnetic materials, in particular anisotropy [5], and is an attractive topic for technical research because of its applications in sensor devices [6]. The MI effect is obtained from the field dependence of the voltage induced across a soft magnetic sample (axial diagonal impedance) when an ac current is flowing through the sample. A method to detect the field-dependent off-diagonal impedance consists in the use of a pick-up coil wound around the sample and is based on the possible existence of a transverse-magnetization process, which appears when the ac driving current induces an ac axial magnetization variation and, hence, a pick-up coil voltage. This effect is referred to as off-diagonal MI. Off-diagonal MI has been measured before in amorphous magnetic ribbons and wires $[7,8]$.

* corresponding author; e-mail: grande@uniovi.es
We report some results comparing the amorphous ribbons of $\mathrm{Co}_{66} \mathrm{Fe}_{4} \mathrm{Ni}_{1} \mathrm{Si}_{15} \mathrm{~B}_{14}$ and $\mathrm{Fe}_{80} \mathrm{~B}_{20}$ with different magnetostriction coefficient, in the as-quenched state and when a transverse magnetic field of $0.07 \mathrm{~T}$ is applied during quenching. The measurements of the off-diagonal impedance spectra allow us to detect small changes of anisotropy on the samples.

\section{Experimental}

Amorphous ribbons with nominal compositions $\mathrm{Co}_{66} \mathrm{Fe}_{4} \mathrm{Ni}_{1} \mathrm{Si}_{15} \mathrm{~B}_{14}$ and $\mathrm{Fe}_{80} \mathrm{~B}_{20}$ were produced by melt spinning. Some of them were quenched while a transverse magnetic field of $0.07 \mathrm{~T}$ was applied along the ribbon plane, known as field quenched samples (fq), compared with the as quenched samples (aq). The ribbons are $10 \mathrm{~cm}$ long. All Fe-rich samples are $29 \mu \mathrm{m}$ thick and $5 \mathrm{~mm}$ wide. The aq Co-rich sample has a $25 \mu \mathrm{m}$ thickness and a $2.5 \mathrm{~mm}$ width. The fq Co-rich sample is $35 \mu \mathrm{m}$ thick and $5.5 \mathrm{~mm}$ wide.

The surface domain structure of the samples was obtained by the Bitter technique. The off-diagonal component of impedance was measured from the voltage induced in a pick-up coil wounded around the sample. The pick-up coil had 400 turns and was $34 \mathrm{~mm}$ long. The samples are connected in series with the circuit using silver conductive paint. An ac current $I_{\mathrm{ac}}$ of $8 \mathrm{~mA}$ rms was provided for frequencies in the $100 \mathrm{kHz}-2 \mathrm{MHz}$ range, while an axial magnetic field applied along the sample's longitudinal direction created by a Helmholtz coil was changed from -100 to 100 Oe. The real and imaginary components of the induced voltage are collected by a lock-in 
amplifier. From the measured voltage amplitude, the off-diagonal impedance $Z_{\mathrm{c}}$ was calculated as $Z_{\mathrm{c}}=\mathrm{V}_{\mathrm{c}} / I$.

\section{Results and discussion}

Figure 1 shows the domain structure of the studied ribbons obtained by the Bitter technique. An obvious change on the surface domain structure can be observed in the $\mathrm{Fe}_{80} \mathrm{~B}_{20}$ sample. For the aq sample, domain patterns exhibit a maze configuration and zigzag walls in regions with perpendicular anisotropy to the ribbon plane, due to the compression stresses developed during the quenching process. Out of these regions the magnetization lies in the ribbon plane with different orientations. Domain patterns are not observed on the field quenched ribbon surface as a consequence of the magnetization direction, that lies in the ribbon plane throughout the entire sample. Both Fe-based samples have a magnetostriction of the order of $+10^{-5}$ [8]. The considerably different domain structure of the ribbons surface is a consequence of the transverse anisotropy component induced on the fq sample during the quenching procedure [9]. However, there are no detectable changes on the Co-rich ribbons surfaces, where domain patterns are not observed for aqor fq samples. Opposite to what happens in the large positive magnetostrictive Fe-rich samples, in the negative magnetostrictive (around $-10^{-6}$ ) Co-rich ribbons the internal stresses induced during the quenching procedure cause the magnetization to lie mainly in the ribbon plane.
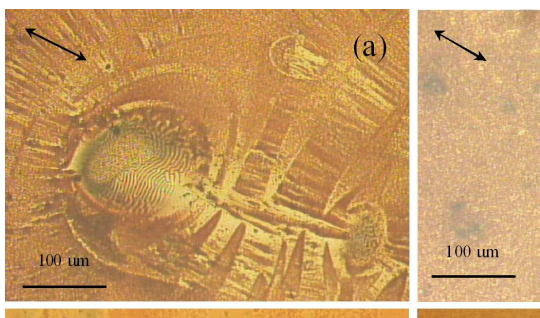

(c)
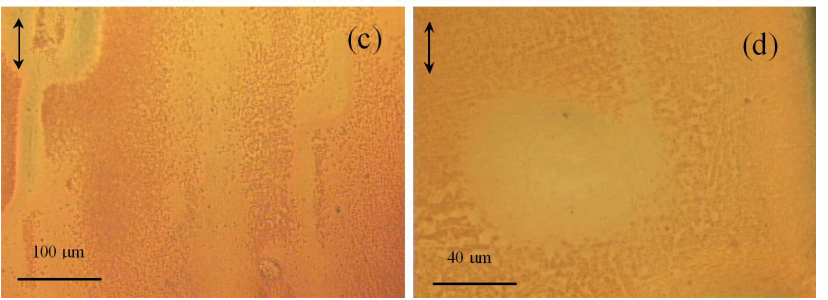

Fig. 1. Domain patterns for $\mathrm{Fe}_{80} \mathrm{~B}_{20}$ ribbons: (a) as-quenched and (b) field-quenched, and for $\mathrm{Co}_{66} \mathrm{Fe}_{4} \mathrm{Ni}_{1} \mathrm{Si}_{15} \mathrm{~B}_{14}$ ribbons: (c) as-quenched and (d) field-quenched. The arrow indicates the longitudinal direction of the ribbon.

When a coil is wounded around the sample to collect the induced voltage, i.e., the off-diagonal impedance component, a resonance may be obtained for some frequency. In our case, this frequency is obtained in our measuring frequency range $(1.3 \mathrm{MHz}$ in the empty coil). The resonance frequency depends not only on the particular coil employed, but also on the sample's transverse permeability, due to the fact that the sample is transversally magnetized by the flowing ac current. The existence of this resonance and its behaviour for different samples and magnetic field provides us with some information about the magnetic state of the samples.

The off-diagonal spectra for the $\mathrm{Fe}_{80} \mathrm{~B}_{20}$ ribbons are represented in Fig. 2 for the aq (Fig. 2a) and fq (Fig. 2b) samples. The values of both real and imaginary parts $\left(R_{\mathrm{c}}\right.$ and $X_{\mathrm{c}}$, respectively) of the off-diagonal impedance are enhanced when a 100 Oe magnetic bias field is applied in the case of the aq sample. The same behaviour can be seen in the fq sample and it is related to the diminishing transverse permeability, as a consequence of the bias field effect. It can be observed in Figs. 2a and b how the resonance frequency decreases at $H=0$ Oe with the change of sample, from $1538 \mathrm{kHz}$ to $398 \mathrm{kHz}$, due to the increase in transverse permeability of the fq sample, as can be seen in the domain structures in Fig. 1. When the saturating magnetic field is applied, the position of the resonance frequency is similar for both cases, as it can be explained by a small and similar value of the transverse permeability.

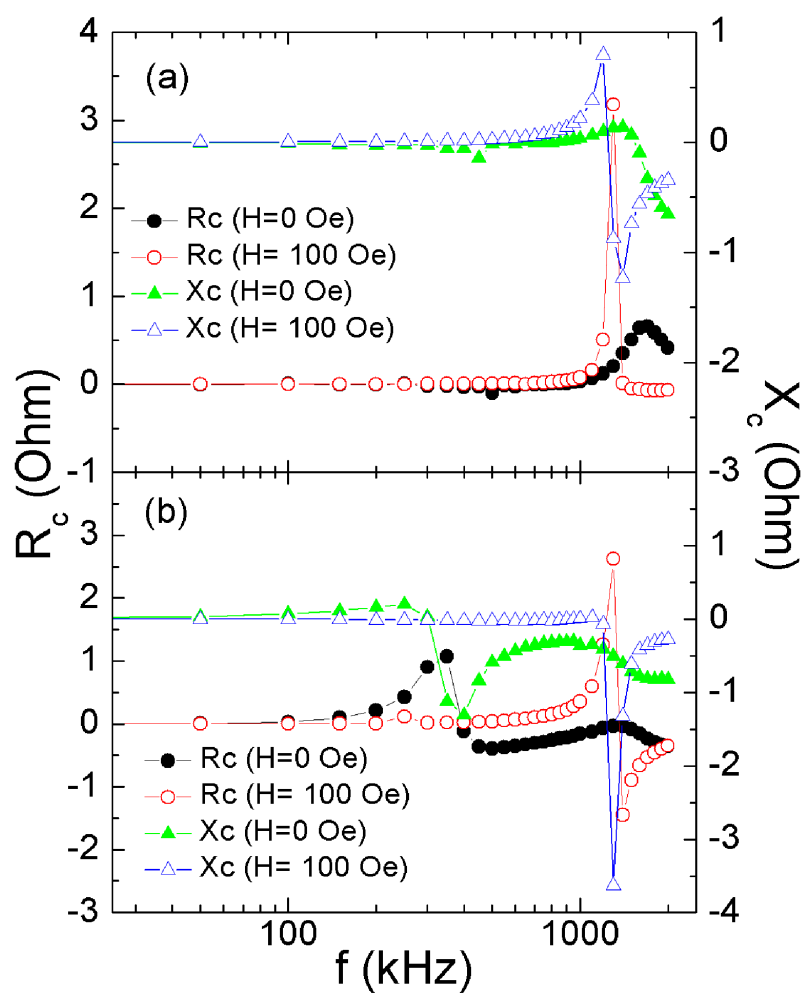

Fig. 2. Off-diagonal MI spectra for: (a) as-quenched and (b) field-quenched $\mathrm{Fe}_{80} \mathrm{~B}_{20}$ ribbons with and without a magnetic bias field of 100 Oe.

Similar results are observed in Fig. 3 for the Co-rich ribbons. The resonance frequency for the $H=0$ Oe case decreases from $463 \mathrm{kHz}$ to $247 \mathrm{kHz}$ due to the increase of transverse permeability produced by the field quenching 


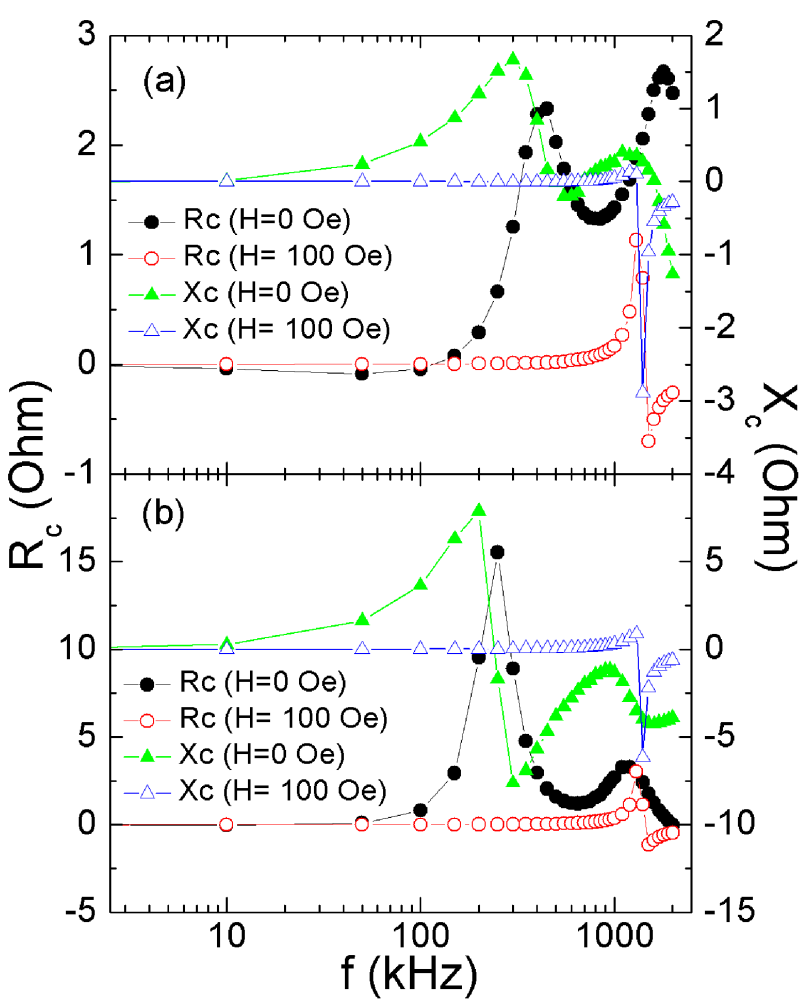

Fig. 3. Off-diagonal spectra for: (a) as-quenched and (b) field-quenched $\mathrm{Co}_{66} \mathrm{Fe}_{4} \mathrm{Ni}_{1} \mathrm{Si}_{15} \mathrm{~B}_{14}$ ribbons with and without a 100 Oe bias field.

procedure. When the 100 Oe bias field is applied longitudinally, the transverse permeability decreases, increasing the resonance frequency. Figure 3 shows two different peaks in the $H=0$ Oe cases. This might be due to the frequency dependence of permeability, which would produce a complex behaviour of off-diagonal impedance and resonance frequency. Further work is being performed in order to clarify the resonance frequency dependence on permeability.

\section{Conclusions}

The comparison of the field-quenching procedure on two types of samples with different composition $\mathrm{Co}_{66} \mathrm{Fe}_{4} \mathrm{Ni}_{1} \mathrm{Si}_{15} \mathrm{~B}_{14}$ and $\mathrm{Fe}_{80} \mathrm{~B}_{20}$ is clearly remarked on the domain patterns obtained by the Bitter technique. Zigzag domain walls are not observed for the field- -quenched Fe-rich sample as a consequence of the transverse magnetic anisotropy contained in the ribbon plane induced on it during the fabrication process. No domain structure is observed on the surface of the Co-rich samples. The negative magnetostriction coefficient gives an explanation about the absence of the regions with perpendicular magnetization orientation to the ribbon plane.

The existence of a resonance frequency in our measuring circuit turns out to be an advantage in order to study the magnetic state of the samples: the resonance frequency at $H=0$ Oe shows a large variation between the aq and fq samples, as large as $1140 \mathrm{kHz}$ for the $\mathrm{Fe}_{80} \mathrm{~B}_{20}$ samples and $214 \mathrm{kHz}$ for the $\mathrm{Co}_{66} \mathrm{Fe}_{4} \mathrm{Ni}_{1} \mathrm{Si}_{15} \mathrm{~B}_{14}$ ones, as a consequence of their different permeability values. These large changes reveal the interest to further develop this simple technique.

\section{Acknowledgments}

This work has been supported by the Spanish MEC under projects MAT2009-13108-C02-01 and MAT2009-13108-C02-02. FICYT is acknowledged by T. Sánchez (BP07-075) for her Ph.D. grant.

\section{References}

[1] R. Hasegawa, J. Magn. Magn. Mater. 215-216, 240 (2000).

[2] G. Kumar, M. Ohnuma, T. Furubayashi, T. Ohkubo, K. Hono, J. Non-Cryst. Solids 354, 882 (2008).

[3] M. Tejedor, J.A. García, J. Carrizo, L. Elbaile, J.D. Santos, Appl. Phys. Lett. 82, 937 (2003).

[4] M. Tejedor, B. Hernando, M.L. Sánchez, V.M. Prida, P. Gorria, J. Non-Cryst. Solids 287, 396 (2001).

[5] M. Knobel, M. Vázquez, L. Kraus, in: Handbook of Magnetic Materials, Ed. K.J.H. Buschow, Elsevier, New York 2002.

[6] L.V. Panina, K. Mohri, Appl. Phys. Lett. 65, 1185 (1994).

[7] N.A. Buznikov, C. Kim, C. Kim, L. Jin, S. Yoon, J. Appl. Phys. 98, 113908 (2005).

[8] T. Sánchez, P. Álvarez, J. Olivera, M.J. Pérez, F.J. Belzunce, J.D. Santos, J.L. Sánchez, Ll., M.L. Sánchez, P. Gorría, B. Hernando, J. Non-Cryst. Solids 353, 914 (2007).

[9] M. Tejedor, J.A. García, J. Carrizo, L. Elbaile, J.D. Santos, J. Appl. Phys. 96, 5658 (2004). 